7 pages, no figures, submitted to Classical and Quantum Gravity

Report-No: BNU-03-66

\title{
Sommerfeld's quantum condition of action and the spectra of Schwarzschild black hole
}

\author{
L. Liu* and S. Y. Pei ${ }^{\dagger}$ \\ Department of physics, Beijing Normal University
}

\begin{abstract}
If the situation of quantum gravity nowadays is nearly the same as that of the quantum mechanics in it's early time of Bohr and Sommerfeld, then a first step study of the quantum gravity from Sommerfeld's quantum condition of action might be helpful. In this short paper the spectra of Schwarzschild black hole(SBH) in quasi-classical approach of quantum mechanics is given. We find the quantum of area is $\frac{8 \pi}{3} l_{p}^{2}$, the quantum of entropy is $\frac{2 \pi}{3} k_{B}$ and the Hawking evaporation will cease as the black hole reaches its ground state $m=\frac{1}{2 \sqrt{3}} m_{p}$.
\end{abstract}

*Electronic address: liuliao1928@yahoo.com.cn

$\dagger$ Electronic address: psy@bnu.edu.cn 
Several different approaches to quantum gravity have been proposed in the recent years. Among them, people believe that loop quantum gravity [LQG] might be a viable one to quantum gravity[1]. However there are several discrepancies with it[2]. The first one, for example, is the appearance of an undetermined free parameter - the so-called Immirziparameter - which plaques with the overall important coefficient " one quarter" in classical black hole physics. The second one is that it has no reasonable low energy and high energy approximation, for example, the low energy approximation of LQG is not the Einstein's general relativity, which has a sounded experimental support now. And LQG in the Planck regions agrees well with Einstein's general relativity, though we have no reasons to believe it. So it seems, we are now in the time as the old quantum physics was in the Bohr-Sommerfeld's time. Therefore a similar first step treatment of quantum gravity by using Sommerfeld's quantum condition of action might be a viable road to future quantum gravity.

\section{ACTION VARIABLE, ACTION AND HAMILTON}

As is known in classical mechanics, one can define a quantity called action variable for a single periodic system[3],

$$
I_{v}=\oint p d q
$$

which is really the area of phase space occupied by the system in one periodic motion. We remind there is an important relation between the action I and the action variable $\mathrm{I}_{v}$ of the single periodic motion, i.e[3][4].

$$
I=I_{v}-\oint H d t
$$

where $\mathrm{H}$ is the Hamiltonian of the system. In the so-called $(3+1)$ decomposition of any Einstein gravitational system, we have however two different kinds of Hamiltons, i.e. the one $H\left(h_{i j}, \pi_{i j}\right)$ without Gibbons-Hawkijng surface term that satisfies the famous Hamilton constraint

$$
H\left(h_{i j}, \pi_{i j}\right)=0
$$

the other 


$$
H^{\prime}=H+\alpha
$$

where

$$
\alpha=\lim _{r \rightarrow \infty} \int_{s}\left(\frac{\partial h_{i j}}{\partial x^{j}}-\frac{\partial h_{j j}}{\partial x^{i}}\right) r^{i}
$$

is just the ADM mass $m$ for asymptotic flat $\mathrm{SBH}$. We remind $h_{i j}$ is the induced metric of the 3-D space-like hypersuface and $\pi_{i j}$ is the canonical conjugated momentum of $h_{i j}$. The important thing in (4) we would like to emphasize is that the Hamilton $H^{\prime}$ of any classical Einstein gravitational system with asymptotic flateness is always the ADM mass $m$ of the system. So an important result is for any Einstein single periodic gravitational system with boundary, we have

$$
I=I_{v}-\oint H^{\prime} d t=\oint p d q-m \oint d t=\oint p d q-m \mathbf{T}
$$

where $\mathbf{T}$ is the perieod of the single periodic motion, $\mathrm{m}$ is the ADM mass of the system.

\section{SOMMERFELD'S QUANTUM CONDITION OF ACTION AND THE SPEC-}

\section{TRA OF SBH}

Early in the year of 1916, A Sommerfeld successfully put forth the so-called Sommerfeld's quantum condition of action[3]

$$
\oint p d q=n h
$$

in order to solve the spectra problem in atom physics. Landau \& Lifshitz derived the Sommerfield's quantum condition for the one dimensional periodic motion of a particle by using the quasi-classical method and get[6]

$$
\oint p d q=2 \pi \hbar\left(n+\frac{1}{2}\right)
$$

that is, for any cyclic motion the action variable $I_{v}$ of the system is quantized.

Now we try to apply this principle to SBH. As is known, the Euclidean Kruskal section of $\mathrm{SBH}$ is a cyclic or single periodic system, whose metric reads 


$$
d s^{2}=\frac{32}{r} m^{3} \exp \left\{-\frac{r}{2 m}\right\}\left(d T^{2}+d R^{2}\right)+r^{2} d \Omega_{2}^{2}, \quad(r>2 m)
$$

where

$$
\begin{aligned}
i T & =\left(\frac{r}{2 m}-1\right)^{\frac{1}{2}} \exp \left\{\frac{r}{4 m}\right\} \sin \left(\frac{\tau}{4 m}\right), \\
R & =\left(\frac{r}{2 m}-1\right)^{\frac{1}{2}} \exp \left\{\frac{r}{4 m}\right\} \cos \left(\frac{\tau}{4 m}\right)
\end{aligned}
$$

Obviously, both $T$ and $R$ in the parametric form (10) and (11) are periodic function of $\tau$ with period $8 \pi \mathrm{m}$. Now we would like to emphasize, that this peculiar property of EuclideanKruskal metric is very important to reveal the thermodynamics of SHB. We shall see now in the following, that this is also of key importance for the recognition of it's quantum property.

From classical general relativity, we know that the area $A$ of the event horizon of SBH and it's action $I$ are

$$
A=16 \pi m^{2}(G=C=1)
$$

and

$$
I=4 \pi m^{2}=\oint p d q-8 \pi m^{2}(G=C=\hbar=1, \mathbf{T}=8 \pi m \text { in eq }(4))
$$

respectively.

Here we note, for the vacuum Euclidean Kruskal section the volume integral of it's action is zero, so the contribution to action comes only from the Gibbons-Hawking's surface term which is $4 \pi m^{2}$. From (12) and (13) the variation $\triangle A$ of $A$ and $\triangle I$ of $I$ have the relation

$$
\triangle A=4 \triangle I(G=C=\hbar=1)
$$

Now after noting for SHB $H^{\prime}=m, I=I_{v}-8 \pi m^{2}$, we can apply Sommerfeld's quantum condition (8) to (12) and (13) and get the spectrum of action $I$, the spectrum of area $A$ of event horizon and the spectrum of entropy $S$ of SBH as follows

$$
I=4 \pi m^{2}=2 \pi \hbar(n+1 / 2)-8 \pi m^{2}
$$


or

$$
\begin{gathered}
m^{2}=\frac{1}{6}\left(n+\frac{1}{2}\right) m_{p}^{2} \\
A=16 \pi m^{2}=\frac{8 \pi}{3}(n+1 / 2) l_{p}^{2}
\end{gathered}
$$

and

$$
S=\frac{1}{4} A
$$

where

$$
I=S=\frac{1}{4} A
$$

.The minimum variation or quantum of the area of the event horizon $\delta A$, quantum of the entropy $\delta S$ and quantum of the mass $\delta m$ of SBH are

$$
\begin{gathered}
\delta A=\frac{8 \pi}{3} l_{p}^{2} \\
\delta S=\frac{2 \pi}{3} k_{B}
\end{gathered}
$$

and

$$
\delta m=\frac{1}{12} \frac{m_{p}^{2}}{m(T)}
$$

respectively. Where $l_{p}=\left(G \hbar C^{-3}\right)^{\frac{1}{2}}$ is the Planck length, $l_{p}^{2}=G \hbar C^{-3}$ is the Planck area, $m_{p}=\left(G^{-1} \hbar C\right)^{\frac{1}{2}}$ is the Planck mass and $\mathrm{m}(T)$ is the mass of SBH at temperature $T$.

It seems our area quantum (20) only refers to the event horizon. We have no reason to infer that this is a general result to all area of any surface. Let's point out, our result of the quantum area (20) is different from the recent value of $4 \sqrt{3} l_{p}^{2}$ by Dreyer and Motl[2]. The total mass-energy loss of a SBH via Hawking evaporation in temperature $T$ is

$$
\int \rho(\nu, T) d \nu=\int \frac{8 \pi h \nu^{3}}{\exp \left(\frac{h \nu}{k T}\right)-1} d \nu \equiv E(T)
$$

From (20) and (23) we obtain the quantum of mass loss(QML) of a SBH via Hawking evaporation at temperature $\mathrm{T}$

$$
\triangle m=E(T)=\frac{1}{12} \frac{m_{p}^{2}}{m(T)}
$$


If the black hole mass $m(T)$ has a lowest limit $\frac{1}{2 \sqrt{3}} m_{p}$ (see Eq. (25)), then the QML $\triangle M=E(T)$ has an upper limit $\triangle m=\frac{1}{2 \sqrt{3}} m_{p}$.

\section{DISCUSSION:}

From (15 ) and (17) we see $\mathrm{n}=0$ should correspond to the ground state of SBH. It is easy to show the ground state mass $\mathrm{m}_{G}$ of $\mathrm{SBH}$ is

$$
m_{G}=\frac{1}{2 \sqrt{3}} m_{p}
$$

It seems, there is no way to decrease the mass of a SBH under it's ground state mass $m_{G}$. Therefore even Hawking evaporation will cease as a SBH approaches it's ground state! That means, no loss of quantum coherence and no violation of unitarity in the last stage of evolution of a black hole! In a word, the long unsolved information puzzle doesn't exist at all[7]! This is a very interesting result from our quasi-classical quantum mechanic approach.

\section{Acknowledgments}

We thank prof J. Y. Zhu for let us know the papers in ref[2] and wish to thank Prof. Z. Zhao and Dr H. Y. Wang for helpful discussions.

[1] Carlo Rovelli, Peush Upadhya, Loop quantum gravity and quanta of space: a primer ar Xiv: gr-qc/980679

[2] Lubos Motl, An analytical computation of asymptotic Schwarzschild quasinormal frequencies,ar Xiv gr-qc/0212096, Olaf Dreyer, Quasinormal Modes, the Area Spectrum, and Black Hole Entropy ar Xiv gr-qc/0211076

[3] T Y Wu, Classical Dynamics (in Chinese), A Sommerfeld, Ann Physik 51;1(1916)

[4] L D Landau and E M Lifshitz Mechanics Third Edition Pergamon Press 1976

[5] R Wald, General Relativity, E2 P469, The University of Chicago Press 1984

[6] L D landau and E M Lifshitz, Quantum Mechanics ( Non-Relativity Theory) Pergamon Press, 1958 
[7] S W Hawking Phys Rev 14D(1976)2460 